Supporting Information

\title{
Enhanced Plasmonic Photocatalysis through Synergistic Plasmonic-Photonic Hybridization
}

Qinglan Huang, ${ }^{1,2}$ Taylor D. Canady, ${ }^{2,3}$ Rohit Gupta, ${ }^{4}$ Nantao Li, ${ }^{1,2}$ Srikanth Singamaneni, ${ }^{4}$ and Brian T. Cunningham ${ }^{1,2,3,5, *}$

${ }^{1}$ Department of Electrical and Computer Engineering, University of Illinois at Urbana-Champaign, Urbana, IL 61801, United States;

${ }^{2}$ Holonyak Micro and Nanotechnology Laboratory, University of Illinois at Urbana-Champaign, Urbana, IL 61801, United States;

${ }^{3}$ Carl R. Woese Institute for Genomic Biology, University of Illinois at Urbana-Champaign, Urbana, IL 61801, United States;

${ }^{4}$ Department of Mechanical Engineering \& Materials Science, Washington University in St. Louis, St. Louis, MO 63130, United States;

${ }^{5}$ Department of Bioengineering, University of Illinois at Urbana-Champaign, Urbana, IL 61801, United States.

*Corresponding author: bcunning@illinois.edu 


\section{Section S1: Materials and Methods}

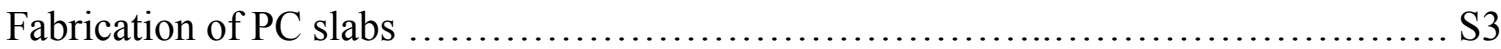

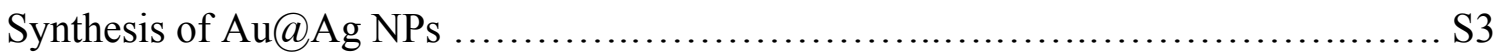

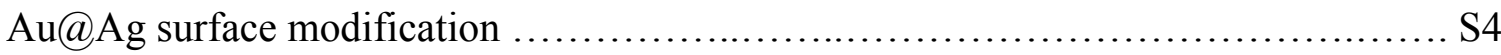

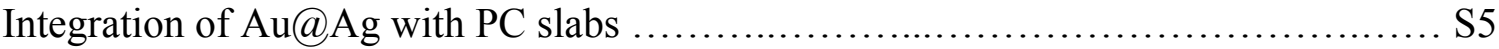

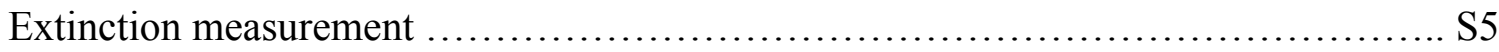

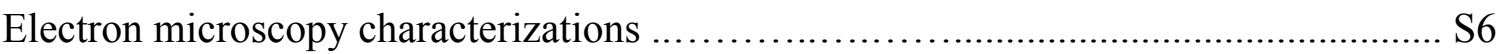

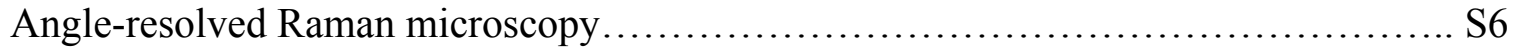

\section{Section S2: Numerical Simulations}

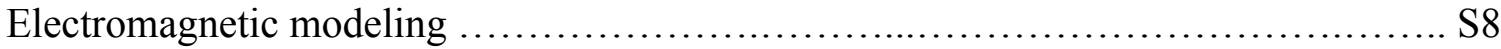

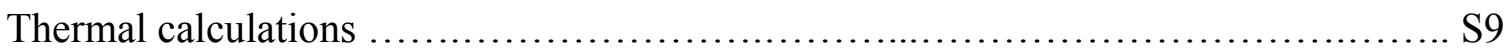

\section{Section S3: Additional Data}

Absorption enhancement ......................................................... S11

SERS of 4-NTP as a function of incidence angles ................................ S13

Power dependence of hot-electron chemistry ....................................... S13

$\mathrm{Au} @ \mathrm{Ag}$ after hot-electron chemistry ............................................... 14

Supplementary References ...................................................... S15 


\section{Section S1: Materials and Methods}

\section{Fabrication of PC slabs}

The PC fabrication started from subsequent deposition of a 10 -nm-thick $\mathrm{Al}_{2} \mathrm{O}_{3}$ etch stop layer and a 130-nm $\mathrm{SiO}_{2}$ thin film onto a 200-mm-diameter glass substrate (Corning, $0.7 \mathrm{~mm}$ Eagle XG display grade glass). Then, the subwavelength grating structures were patterned by large-area deep ultraviolet (DUV) lithography followed by dry etch performed by a foundry (Moxtek, Inc. Orem, UT). The wafer was then diced into small chips $(1 \mathrm{~cm} \times 1 \mathrm{~cm})$. A high-refractive-index $\mathrm{TiO}_{2}$ thin film was deposited onto the patterned substrate using a sputter (Kurt J. Lesker PVD 75). Finally, a 5-nm-thick $\mathrm{SiO}_{2}$ thin film was sputtered onto the surface to electronically isolate $\mathrm{TiO}_{2}$ and subsequently-added metal nanoparticles (NPs).

\section{Synthesis of Au@Ag NPs}

$\underline{\text { Materials }}$ Ascorbic acid (AA, $\geq 99.0 \%$ ), gold(III) chloride trihydrate $\left(\mathrm{HAuCl}_{4} \cdot 3 \mathrm{H}_{2} \mathrm{O}\right.$, $\geq 99.9 \%$, sodium borohydride $\left(\mathrm{NaBH}_{4}, 98 \%\right)$, silver nitrate $\left(\mathrm{AgNO}_{3},>99 \%\right)$, Hydrochloric acid $(\mathrm{HCl})$, cetyltrimethylammonium bromide $(\mathrm{CTAB}, \geq 99 \%)$, and cetyltrimethylammonium chloride (CTAC, $\geq 98 \%$ ), were obtained from Sigma-Aldrich. All chemicals were used as received without further modifications.

Synthesis of AuNR (Gold nanorods) AuNR (LSPR wavelength $720 \mathrm{~nm}$ ) was prepared by seed mediated method. ${ }^{1,2}$ Gold seed was synthesized by adding $0.6 \mathrm{ml}$ of ice-cold $\mathrm{NaBH}_{4}$ solution (10 mM) (Sigma-Aldrich, 71321) into a solution containing $0.25 \mathrm{ml} \mathrm{HAuCl}$ (10 mM) (SigmaAldrich, 520918) and $9.75 \mathrm{ml} \mathrm{CTAB} \mathrm{(0.1} \mathrm{M)} \mathrm{(Sigma-Aldrich,} \mathrm{H5882)} \mathrm{under} \mathrm{vigorous} \mathrm{stirring} \mathrm{at}$ room temperature for $10 \mathrm{~min}$. The solution color changed from yellow to brown indicating the formation of $\mathrm{Au}$ seed. For the synthesis of AuNR, the growth solution was prepared by the 
sequential addition of aqueous $\mathrm{HAuCl}_{4}(0.01 \mathrm{M}, 2 \mathrm{ml}), \mathrm{CTAB}(0.1 \mathrm{M}, 38 \mathrm{ml}), \mathrm{AgNO}_{3}(0.01 \mathrm{M}$, $0.5 \mathrm{ml}$, Sigma-Aldrich, 204390), HCI (1M, $0.8 \mathrm{ml}$, Sigma-Aldrich, H9892) and ascorbic acid (0.1 M, 0.22ml, Sigma-Aldrich, A92902) followed by gentle inversion to homogenize the solution. The $\mathrm{AgNO}_{3}$ and $\mathrm{HCI}$ volume ratio may vary to obtain the right wavelength. Subsequently, $5 \mu \mathrm{l}$ of the seed solution was added into the growth solution and left undisturbed in dark for 24 hours. AuNR solution was centrifuged at $7000 \mathrm{rpm}$ for minutes to remove the supernatant and the AuNR was re-dispersed into nanopure water to achieve a final extinction $\sim 2.0$.

Synthesis of Au@Ag (Ag cuboid shell with AuNR core) AuNR with LSPR wavelength around $720 \mathrm{~nm}$ was employed as the core for the synthesis of $\mathrm{Au} @ \mathrm{Ag}$ nanostructures. Specifically, $3 \mathrm{ml}$ of once-centrifuged $720 \mathrm{~nm}$ AuNR (extinction 4) was incubated with $8 \mathrm{ml}$ of CTAC (20 $\mathrm{mM})$ at $60{ }^{\circ} \mathrm{C}$ for 20 minutes under stirring. Then, $8 \mathrm{ml}$ of $\mathrm{AgNO}_{3}(4 \mathrm{mM}), 4 \mathrm{ml}$ of CTAC $(20$ $\mathrm{mM})$, and $0.8 \mathrm{ml}$ of ascorbic acid $(0.1 \mathrm{M})$ were added sequentially and the mixture was incubated at $60{ }^{\circ} \mathrm{C}$ for $4 \mathrm{~h}$ under magnetic stirring to form $\mathrm{Au} @ \mathrm{Ag}$. Finally, $\mathrm{Au} @ \mathrm{Ag}$ solution was centrifuged at $6000 \mathrm{rpm}$ and the nanocuboids were redispersed in nanopure water.

\section{Au@Ag surface modification}

To functionalize $\mathrm{Au} @ \mathrm{Ag}$ surface, $100 \mu \mathrm{L}$ of $10 \mathrm{mM}$ ethanolic solution of the corresponding thiols: 4-NTP (Sigma-Aldrich, N27209) or 4-ATP (Sigma-Aldrich, 422967), were added to $1 \mathrm{mLAu@Ag} \mathrm{solution}$ and incubated overnight. The thiols easily replace the CTAC surfactant to form a self-assembled monolayer on the Au@Ag surface. The solution was then centrifuged and redispersed in nanopure water. Red shift in the NP extinction spectra validate the chemisorption of the thiols. 


\section{Integration of Au@Ag NPs with PC slabs}

Freshly prepared solution of thiol-modified Au@Ags $(30 \mu \mathrm{L}$, extinction =1) was drop cast onto an oxygen plasma treated PC surface. The substrate was dried in ethanol vapor, where the Marangoni flow effect prevents the "coffee-stain" rings of aggregated nanoparticles. ${ }^{3}$ As a result, a stable coating of Ag@Au with uniform surface density of $\sim 3 \mu \mathrm{m}^{-2}$ was formed. The NP-PC hybrid substrates were used immediately after preparation.

\section{Extinction measurement}

We characterized the LSPR of the Au@Ag NPs using a UV-vis-NIR spectrophotometer Cary 5000. The extinction spectrum of Au@Ag suspended in solution is shown in Fig. S1. The major peak at $\lambda=592 \mathrm{~nm}$ corresponds to the longitudinal LSPR mode.

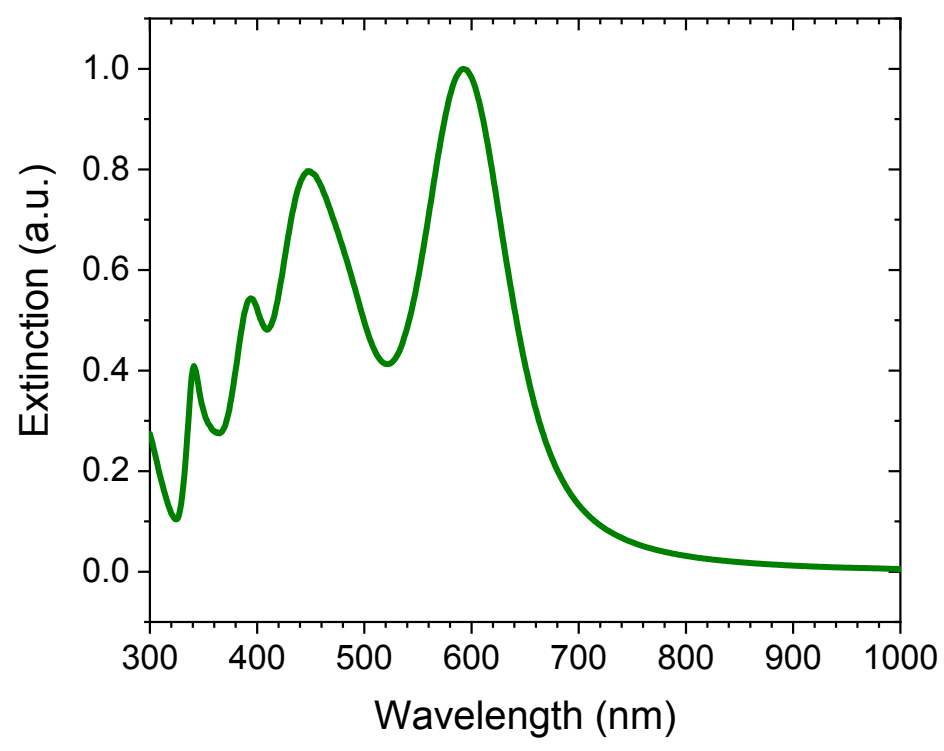

Fig. S1. Extinction spectrum of the as-synthesized Au@Au solution. 
An adjacent high-index dielectric substrate can strongly influence the plasmonic NP. ${ }^{4}$ To correctly measure the LSPR of Au@Ag on a PC surface, we immobilized Agu@Ag NPs onto a $\mathrm{SiO}_{2} / \mathrm{TiO}_{2}$-coated glass substrate to mimic the dielectric environment of a PC surface. The NP coated glass was immersed in water and probed with Cary 5000. A $\mathrm{SiO}_{2} / \mathrm{TiO}_{2}$-coated glass substrate but without NPs served as the reference sample. The measured extinction of Au@Ag on substrate is shown in Fig. 2(e) left panel in the main text. Compared to the LSPR of Au@Ag suspended in solution (Fig. S1), the high-index $\mathrm{TiO}_{2}$ substrate significantly redshifts the LSPR, matching the plasmonic resonance to the PCGR mode at $\lambda=633 \mathrm{~nm}$.

\section{Electron microscopy characterizations}

Transmission electron microscopy (TEM) images of the Au@Ags were collected using a JEOL JEM-2100F field emission microscopy. Scanning electron microscopy (SEM) images of the hybrid sample were obtained using a Hitachi S-4800 field emission SEM. In SEM, gold was sputtered onto the sample for conductivity.

\section{Angle-resolved Raman microscopy}

We used a home-built line-focusing microscope (Fig. S3) to excite the NP-PC hybrid and probe the Raman scattering from the chemical transformation. A $632.8 \mathrm{~nm} \mathrm{HeNe} \mathrm{laser} \mathrm{serves} \mathrm{as}$ the excitation light source. The beam diameter is first expanded to $\sim 1 \mathrm{~cm}$ by two relay lenses (not shown). The linear polarizer sets the incidence field to be transverse magnetic, whereas the half waveplate controls the illumination power reaching the sample surface. A cylindrical lens focuses the expanded incidence beam along a $y$-axis orientated line onto the back focal plane of a long working distance objective (Olympus LMPLFLN $10 \times$, NA 0.25). In $x z$-plane, the focused BFP 
beam is collimated by the objective onto the sample surface. Translational motion of the incident beam focused on the BFP along $x$-axis results in the change of incidence angles. In $y z$-plane, on the other hand, the incoming laser beam is collimated, filling the rear pupil of the objective, and finally focused on the sample surface. The width and length (Gaussian beam waist) of the lineshaped laser focused on the sample surface are measured to be $2 \mu \mathrm{m}$ and $500 \mu \mathrm{m}$, respectively. The Raman scattered light was collected by the objective, passed through the dichroic mirror, and focused by the microscope tube lens onto the slit of the imaging spectrometer (Horiba iHR 550). The Raman spectrum of the illuminated line is displayed onto the CCD (Synapse EMCCD).

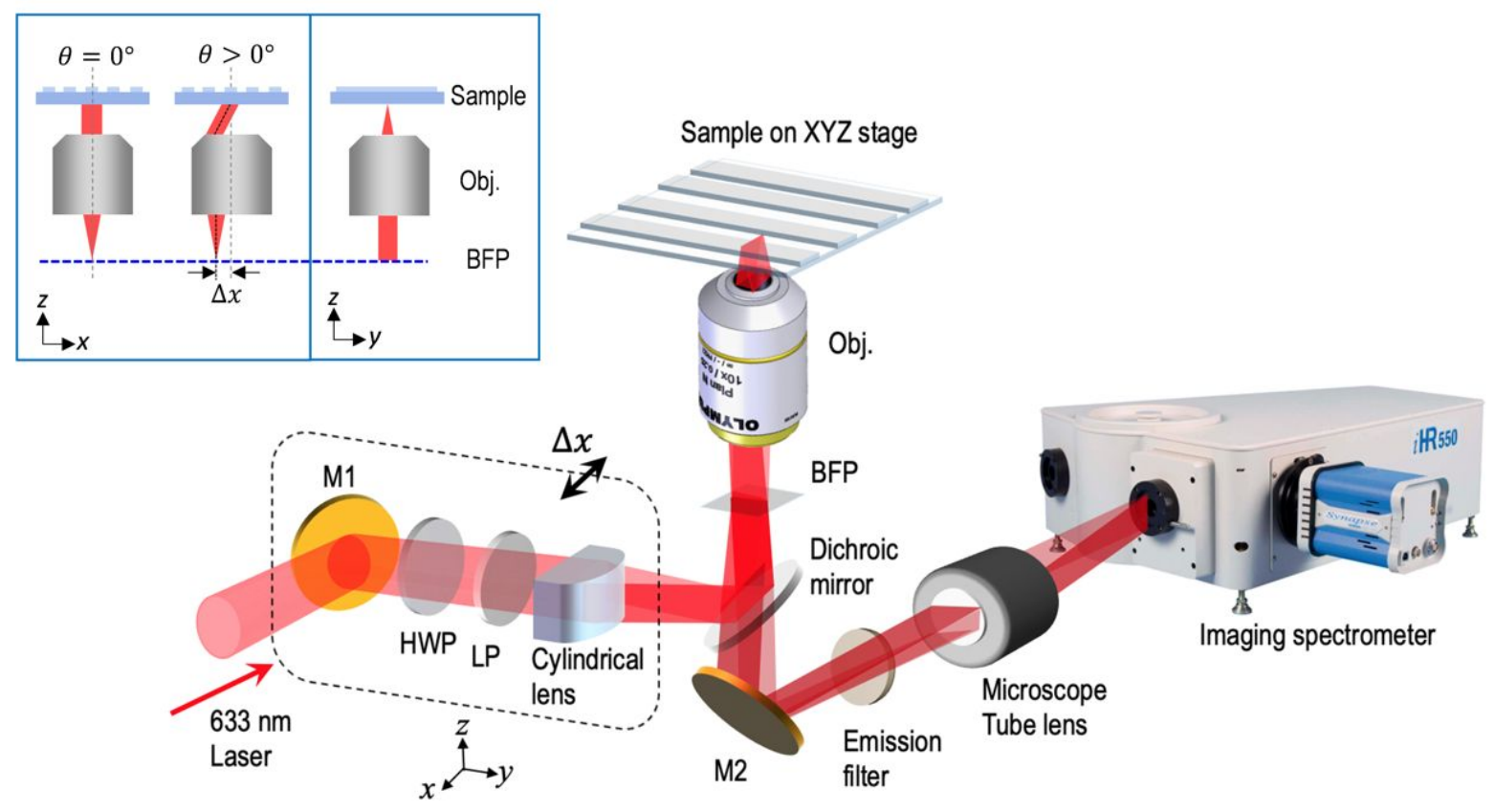

Fig. S2. Schematic diagram of the experimental setup for Raman spectroscopy. Line-focusing Raman microscope with incidence angle tuning capability. M: mirror, HWP: half waveplate, LP: linear polarizer, Obj: objective, BFP: back focal plane. The optics in the round-edged box are mounted on a computercontrolled motion stage which can translate along $x$-axis. Inset: illustration of angle tuning mechanism. 


\section{Section S2: Numerical Simulations}

\section{Electromagnetic Modeling}

We performed 3D electromagnetic simulations using COMSOL Multiphysics software with Wave Optics Module. The simulated physical domain spans three PC lattices in $x$-axis, $1 \mu$ $\mathrm{m}$ in $y$-axis, and $1.25 \mu \mathrm{m}$ in $z$-axis. The geometric parameters of the PC slab $(P=380 \mathrm{~nm}, d=130$ $\mathrm{nm}, t=76.7 \mathrm{~nm}, f_{1}=0.521, f_{2}=0.356, f_{3}=0.45, f_{4}=0.61$, as defined in Fig. 1 (a)) are obtained from SEM. An individual Au@Ag is centered on the ridge of a PC grating and orientated in $x$ axis. The complex structure of $\mathrm{Au} @ \mathrm{Ag}$ (an AuNR core with an Ag cuboid shell) was modeled by considering both the TEM image of the NPs (Fig. 2(a)) and fitting of the simulated LSPR to the experimental results (Fig. 2(e) left panel). In our simulation, silver cuboid shell is set to be $60 \mathrm{~nm}$ long and $40 \mathrm{~nm}$ wide (high), and the length and width of the AuNR core are $42 \mathrm{~nm}$ and $22 \mathrm{~nm}$, respectively.

The refractive indices for gold and silver were taken from Johnson and Christy. ${ }^{5}$ For water, $\mathrm{SiO}_{2}$ and $\mathrm{TiO}_{2}$ that are transparent and weakly dispersive in the red spectral region, we used constant refractive indices $n_{\text {water }}=1.33, n_{\mathrm{SiO} 2}=1.47$ and $n_{\mathrm{TiO} 2}=2.35$. Floquent periodic boundary conditions were set in the $x$ - and $y$ - directions, and PML domains with PEC boundary conditions were imposed in the $z$-direction. Nonuniform mesh was established across the large simulation domain, where extremely fine mesh $(0.35 \mathrm{~nm})$, fine mesh, and coarser mesh were imposed in the peripheral of the Au@Ag, PC slab gratings, and water superstrate/glass substrate, respectively. The mesh setting was validated by a series of convergence tests. The excitation field was sent from the bottom incidence port with transverse magnetic polarization and angle $\theta_{b}=\operatorname{asin}\left(n_{\text {air }}\right.$ * $\left.\sin \theta / n_{\mathrm{SiO} 2}\right)$, where $\theta$ is the incidence angle defined with regard to air.

The absorption power $P_{a b s}$ of the nanoparticle is calculated as: 


$$
P_{a b s}=\int_{\mathrm{Au} @ \mathrm{Ag}} \frac{1}{2} \omega \operatorname{Im}[\varepsilon(\mathbf{r}, \omega)]|\mathbf{E}(\mathbf{r}, \omega)|^{2} \mathrm{~d} \mathbf{r}^{3}
$$

The absorption cross section is obtained as $\sigma_{\mathrm{abs}}=P_{\mathrm{abs}} / P_{\text {inc }} \cdot S_{\text {sim }}$, where $P_{\text {inc }}$ is the incidence power from the port, and $S_{\text {sim }}$ is the area of unit cell $(3 P \times 1 \mu \mathrm{m})$ in the simulation.

a

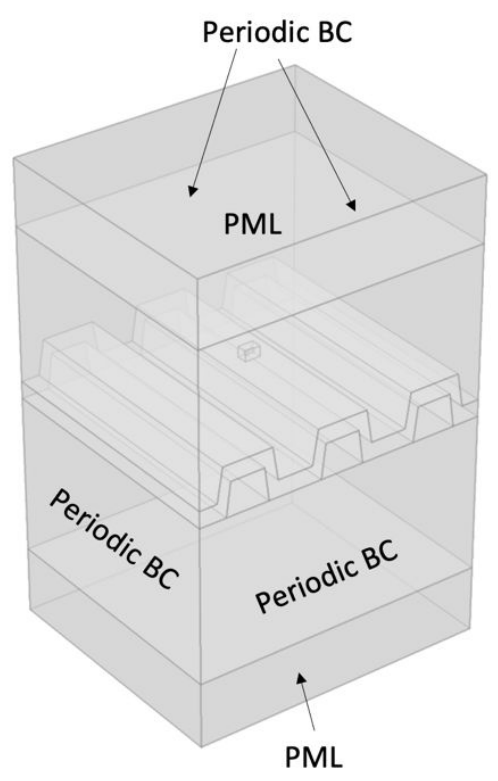

b

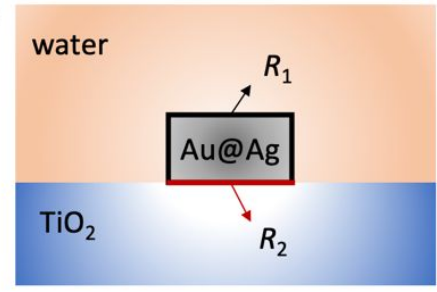

C

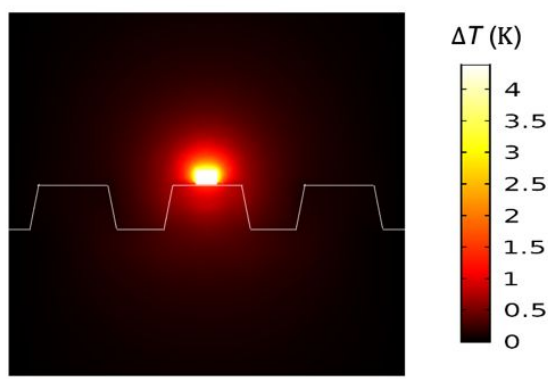

Fig. S3. Comsol modeling method. (a) Simulation domain and boundary conditions for electromagnetic simulation. (b) Boundary conditions for heat transfer modeling. (c) $x-z$ cross section temperature map on PCGR_LSPR hybrid resonance.

\section{Thermal Calculations}

Thermal simulations were performed using the Heat Transfer in Solids Module from COMSOL Multiphysics. The simulation domain is the same as Fig. 3(A), consisting of three PC lattices with a single $\mathrm{Au} @ \mathrm{Ag}$ placed on the ridge center. The steady state temperature field $T$ around the Au@Ag is given by the heat diffusion equation ${ }^{6}$ 


$$
\begin{array}{ll}
\nabla \cdot[\kappa(\mathbf{r}) \nabla T(\mathbf{r})]=-q(\mathbf{r}) & \text { inside the Au@Ag, } \\
\nabla \cdot[\kappa(\mathbf{r}) \nabla T(\boldsymbol{r})]=0 & \text { outside the } \mathrm{Au} @ \mathrm{Ag}
\end{array}
$$

where $\kappa(\mathbf{r})$ is the thermal conductivity and $q(\mathbf{r})$ is the heat density. The function $q(\mathbf{r})$ represents the heat (energy) source generated by light absorption in the materials. Here we assume Au@Ag NP to be the only heat source. Considering that the thermal conductivities of the gold/silver are much greater than those of the surrounding media, it was assumed that the temperature and heat power density are homogeneous inside the $\mathrm{Au} @ \mathrm{Ag}$, and therefore the volumetric heat source of the NP can be approximated by

$$
q(\mathbf{r})=\frac{\sigma_{a b s} I}{V_{\mathrm{Au} @ \mathrm{Ag}}}
$$

where $I$ is the illumination intensity, $\sigma_{a b s}$ is the absorption cross section of the NP, and $V_{\mathrm{Au} @ \mathrm{Ag}}$ is the nanoparticle volume. It is important to note that thermal contact resistance can strongly influence the temperature built up. ${ }^{7}$ To account for this effect, contact resistance $R_{1}=4 \mathrm{~km}^{2} / G W$ and $R_{2}=12 \mathrm{~km}^{2} / G W$ are implemented at the $\mathrm{NP}$ - water and $\mathrm{NP}-\mathrm{TiO}_{2}$ interfaces, respectively ${ }^{7}$ (Fig. S3(B)). Fixed temperature boundary conditions were imposed at the boundaries of the large simulation domain.

The laser power used to stimulate the chemical reaction is measured to be $7 \mathrm{~mW}$. The spot size of the line-shaped illumination beam is measured to be $2 \mu \mathrm{m} \times 500 \mu \mathrm{m}$ (fitted to Gaussian beam waist). Hence the illumination intensity is $I=7 \times 10^{-3} \mathrm{~mW} / \mu \mathrm{m}^{2}$, which is much smaller than that typically used in plasmonic hot carrier chemistry. 8,9 As shown in the main text, $\sigma_{a b s}$ is a function of PCGR — LSPR coupling. To estimate the maximum temperature increase, we utilized the PCGR-enhanced absorption of $\sigma_{a b s}=0.28 \mu \mathrm{m}^{2}$, which is $35 \times$ amplified than that of the solitaryAu@Ag (Fig. 1b in the main text). 
Thermal conductivity of the Au was taken as $318 \mathrm{~W} /(\mathrm{m} \cdot \mathrm{K})$, density as $19320 \mathrm{~kg} / \mathrm{m}^{3}$, and heat capacitance as $129 \mathrm{~J} /(\mathrm{Kg} \cdot \mathrm{K}) .{ }^{10}$ Thermal conductivity of the Ag was taken as $429 \mathrm{~W} /(\mathrm{m} \cdot \mathrm{K})$, density as $10490 \mathrm{~kg} / \mathrm{m}^{3}$, and heat capacitance as $236.91 \mathrm{~J} /(\mathrm{Kg} \cdot \mathrm{K}) .{ }^{10}$ Thermal conductivity of the $\mathrm{TiO}_{2}$ was taken as $8.5 \mathrm{~W} /(\mathrm{m} \cdot \mathrm{K})$, density as $4260 \mathrm{~kg} / \mathrm{m}^{3}$, and heat capacitance as $690.94 \mathrm{~J} /(\mathrm{Kg} \cdot$ K). ${ }^{11}$ The material parameters for glass and water are taken from COMSOL built in material library.

The very low illumination intensity in our experimental setup renders it difficult to measure NP temperature through the anti-Stokes thermometry technique. ${ }^{7,} 12$ However, our simulation gives a temperature rise of $4.3 \mathrm{~K}$ qualitatively matches the literature report. ${ }^{8}$ Considering the PCGR-coupled absorption enhancement effect, the effective illumination intensity in our experiment is approximately $7 \times 10^{-3} \times 35=0.245 \mathrm{~mW} / \mu \mathrm{m}^{2}$. This level of low intensity corresponds to a temperature increase of $<10 \mathrm{~K}$ in Ref. 8 .

\section{Section S3: Additional Data}

\section{Absorption enhancement}

Angle-resolved far-field characterization of hybrid PC structures was performed to demonstrate absorption enhancement effect. ${ }^{13}$ The sample was covered in water and mounted on a motorized rotor with its y-axis aligned to the rotation axis. A collimated beam (area $\sim 5 \mathrm{~mm}^{2}$ ) of white light from a halogen lamp passes through a linear polarizer (TM polarization) and impinges the sample at incidence angle $\theta$. The zeroth-order reflected and transmitted light at a series of incidence angles were collected by collimators and sent to the spectrometer (Horiba iHR 550 equipped with Synapse EMCCD) through optical fibers. The reflection was referenced to a gold mirror. 


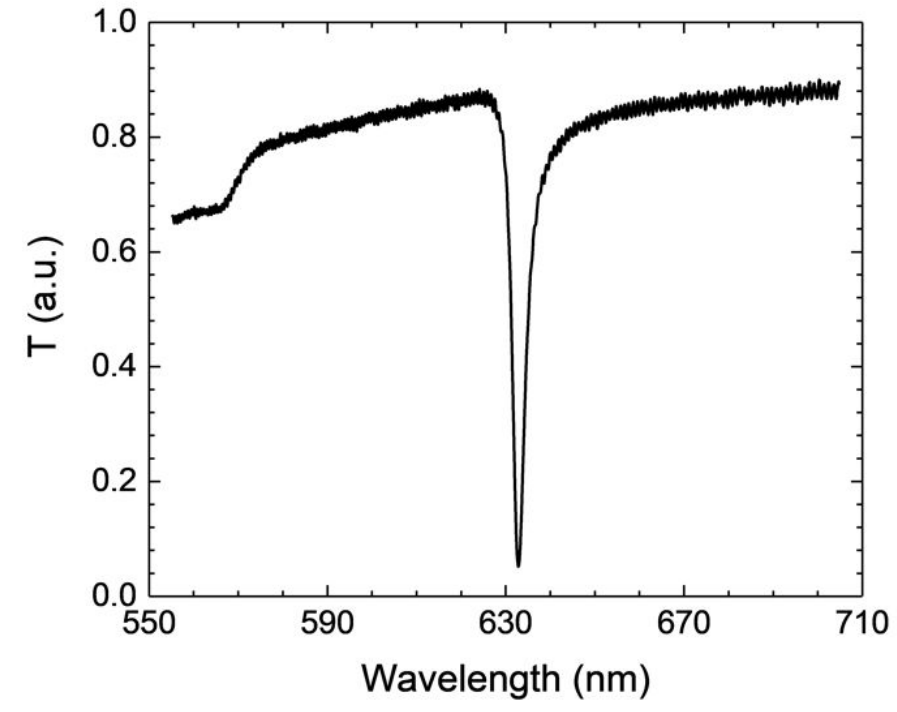

Fig. S4. Transmission spectrum of a bare PC slab at $\theta=3.5^{\circ}$, showing a sharp resonance at $\lambda=$ $633 \mathrm{~nm}$.

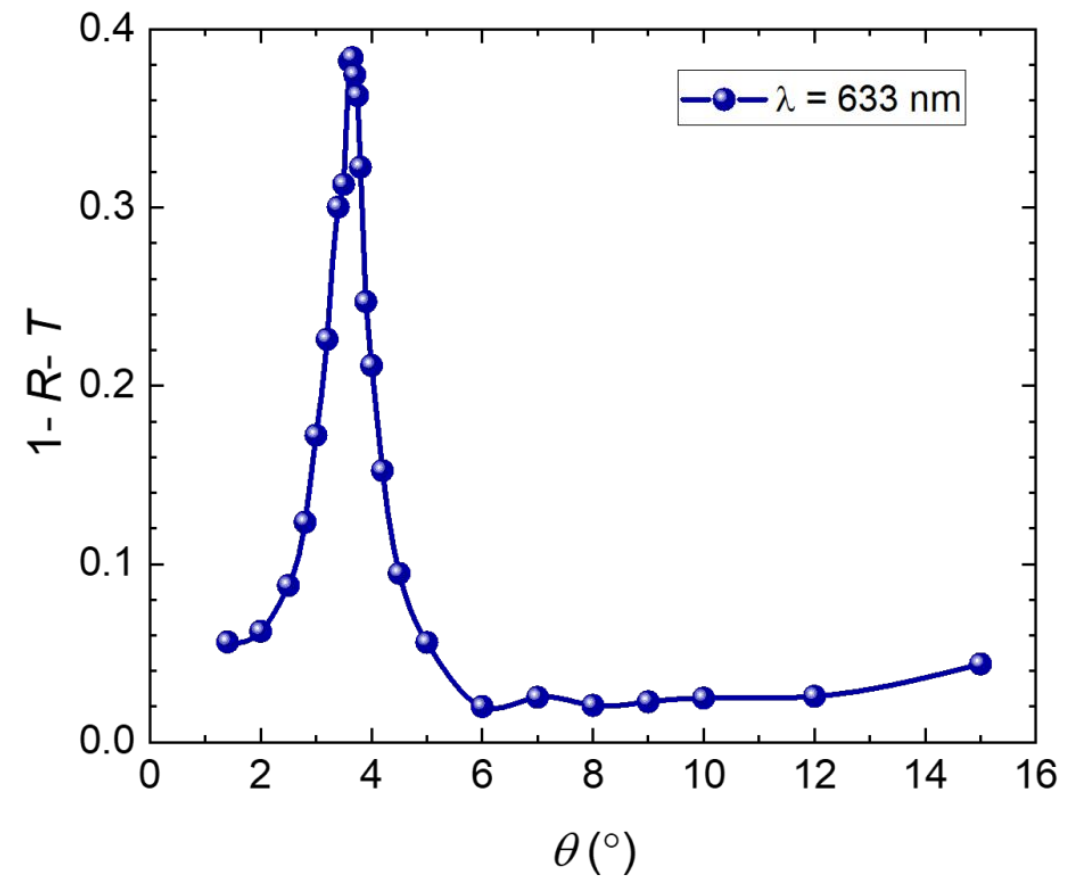

Fig. S5. 1-Reflectance-Transmittance spectrum of the $\mathrm{Au} @ \mathrm{Ag}-\mathrm{PC}$ hybrid sample at $\lambda=633 \mathrm{~nm}$ as a function of incidence angle. 


\section{SERS of 4-NTP as a function of incidence angle}

We measured the SERS spectrum of 4-NTP-modifed Au@Ags placed on a PC slab when the hybrid surface was immersed in water. In absence of $\mathrm{HCl}$, the SERS spectra do not change over time and can therefore serve as a near-field probe. The SERS intensity changes as a function of incidence angle, as shown in Fig. S6. The angle-dependent SERS amplification is in line with the angle-dependent absorption enhancement (Fig. S5), further provide evidence for the PCGRLSPR hybrid enhancement effect.

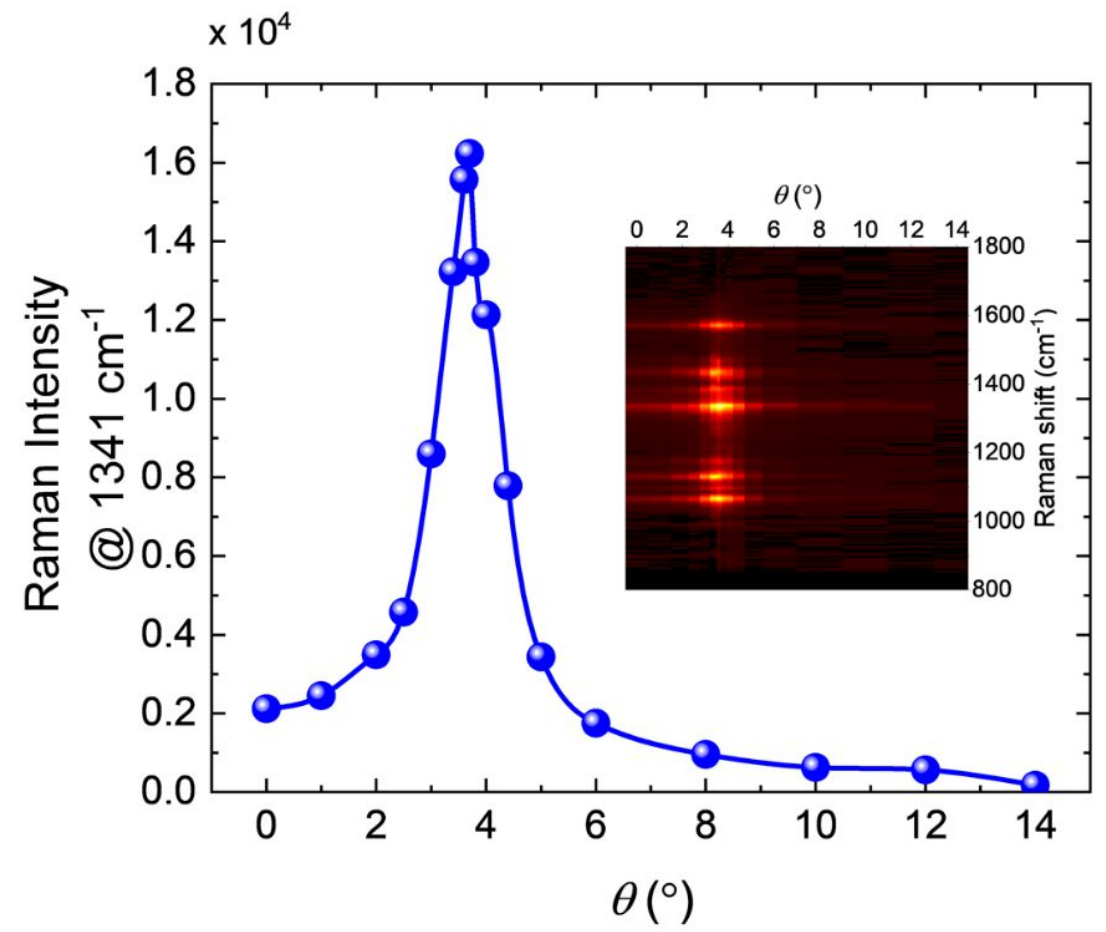

Fig. S6. Evolution of the SERS intensity of 4-NTP molecules (at $1341 \mathrm{~cm}^{-1}$ ) chemisorbed on the Au@AgPC hybrid structure as a function of incidence angle. Inset: SERS spectra of 4-NTP molecules at various incidence angles.

\section{Power dependence of hot electron chemistry}


We investigated the power dependence of the hot-electron driven NTP to ATP conversion with a $633 \mathrm{~nm}$ laser at different powers. A confocal Raman microscope was used to probe the NTP-modified $\mathrm{Au} @ \mathrm{Ag}$ NPs suspended in $0.5 \mathrm{M}$ aqueous solution of $\mathrm{HCl}$. The integration time for each laser power level is $2 \mathrm{~s}$. The conversion rate as a function of laser power is shown in Fig. S7. No reaction was observed when the laser power is below $\sim 2 \mathrm{~mW}$. Above the threshold, the reaction efficiency increases linearly with the laser power.

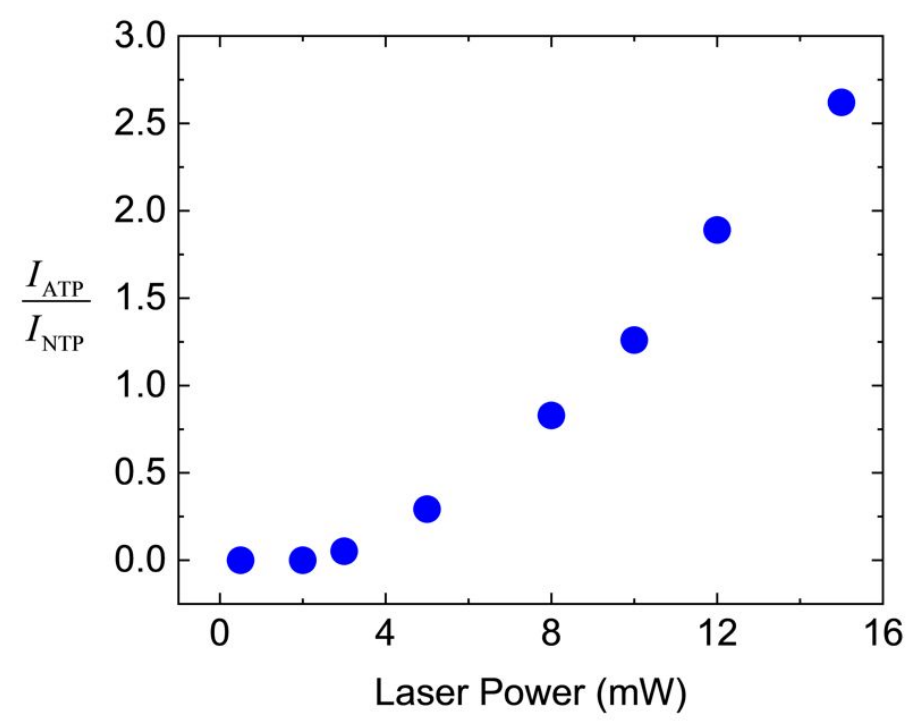

Fig. S7. Power dependence of the hot-electron driven NTP to ATP conversion.

\section{Au@Ag after hot electron chemistry}
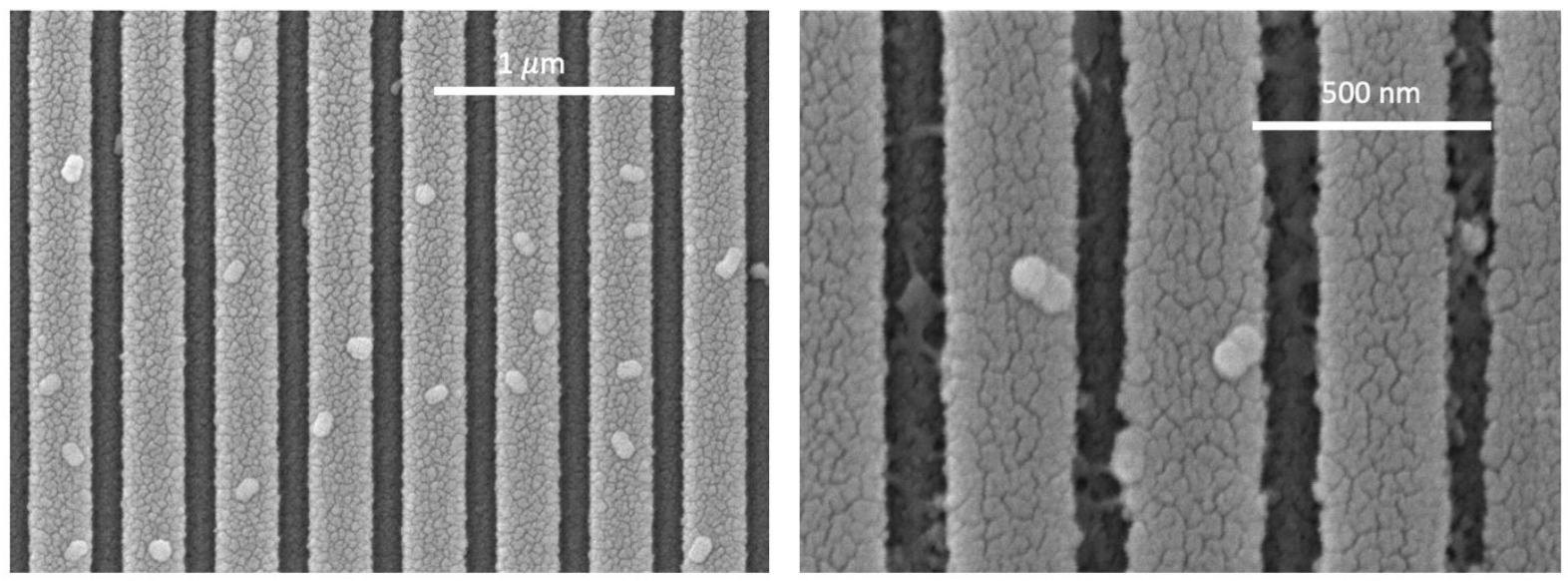
Fig. S8. SEM image of the hybrid sample after hot electron reduction chemistry. There is no substantial morphology change in $\mathrm{Au} @ \mathrm{Ag}$ NPs after the reaction.

\section{Supplementary References}

1. Jana, N. R.; Gearheart, L.; Murphy, C. J. Advanced Materials 2001, 13, (18), 1389-1393.

2. Orendorff, C. J.; Gearheart, L.; Jana, N. R.; Murphy, C. J. Phys Chem Chem Phys 2006, 8, (1), 165-170.

3. Majumder, M.; Rendall, C. S.; Eukel, J. A.; Wang, J. Y. L.; Behabtu, N.; Pint, C. L.; Liu, T.-Y.; Orbaek, A. W.; Mirri, F.; Nam, J.; Barron, A. R.; Hauge, R. H.; Schmidt, H. K.; Pasquali, M. The Journal of Physical Chemistry B 2012, 116, (22), 6536-6542.

4. Knight, M. W.; Wu, Y.; Lassiter, J. B.; Nordlander, P.; Halas, N. J. Nano Letters 2009, 9, (5), 2188-2192.

5. Johnson, P. B.; Christy, R. W. Phys Rev B 1972, 6, (12), 4370-4379.

6. Donner, J. S.; Baffou, G.; McCloskey, D.; Quidant, R. ACS Nano 2011, 5, (7), 5457-5462.

7. Jones, S.; Andrén, D.; Karpinski, P.; Käll, M. ACS Photonics 2018, 5, (7), 2878-2887.

8. Pensa, E.; Gargiulo, J.; Lauri, A.; Schlücker, S.; Cortés, E.; Maier, S. A. Nano Letters 2019, 19, (3), 1867-1874.

9. Cortes, E.; Xie, W.; Cambiasso, J.; Jermyn, A. S.; Sundararaman, R.; Narang, P.; Schlucker, S.; Maier, S. A. Nat Commun 2017, 8.

10. Young, H. D., University physics. Addison-Wesley: 1992.

11. Cahill, D. G.; Allen, T. H. Applied Physics Letters 1994, 65, (3), 309-311.

12. Benz, F.; Schmidt, M. K.; Dreismann, A.; Chikkaraddy, R.; Zhang, Y.; Demetriadou, A.; Carnegie, C.; Ohadi, H.; de Nijs, B.; Esteban, R.; Aizpurua, J.; Baumberg, J. J. Science 2016, 354, (6313), 726 .

13. Huang, Q.; Cunningham, B. T. Nano Letters 2019, 19, (8), 5297-5303. 
